CASO CLÍNICO

\title{
Síndrome de Bertolotti. Una causa importante de dolor lumbar
}

\author{
Bertolotti syndrome. An important cause of lumbar spine pain
}

Clara A. Vidaurre-Herrera* y Laura Quiroz Rojas

Departamento de Resonancia Magnética, Grupo CT Scanner; Ciudad de México, México

\section{RESUMEN}

La presencia de megaapófisis en «vértebras de transición lumbosacra» cuando se asocian con dolor crónico de espalda baja componen el síndrome de Bertolotti, el cual debe considerarse en pacientes adultos jóvenes. Una vértebra transicional modifica la anatomía y la biomecánica normal de la columna vertebral, y causa alteraciones en distintos niveles, principalmente facetario, sacroilíaco o discal.

Palabras clave: Vértebra transicional. Dolor lumbar. Bertolotti.

\section{ABSTRACT}

The presence of megaapophysis of the so-called «lumbosacral transition vertebrae» when associated with chronic low back pain make up the «Bertolotti syndrome», which should be considered in young adult patients. A transitional vertebra modifies the anatomy and normal biomechanics of the spine, causing alterations at different levels, principally faceted, sacroiliac or discal.

Key words: Transitional vertebra. Back pain. Bertolotti.

\section{INTRODUCCIÓN}

En 1917, Mario Bertolotti describió la presencia de una variante anatómica de las vértebras lumbares más caudales, con un proceso

\section{Correspondencia:}

*Clara A. Vidaurre-Herrera

E-mail: alevidarx@gmail.com

2604-2053/C 2019 Sociedad Mexicana de Radiologia e Imagen, AC. Publicado por Permanyer. Este es un artículo open access bajo la licencia CC BY-NC-ND (http://creativecommons.org/licenses/by-nc-nd/4.0/). transverso anormalmente agrandado que se articulaba o fusionaba de manera uni o bilateral con la base del sacro o la cresta ilíaca. Esta megaapófisis de las llamadas «vértebras de transición lumbosacra» (VTLS) cuando se asocia a dolor progresivo de la espalda baja 
o ciática componen el síndrome de Bertolotti $(\mathrm{SB})^{1}$.

La prevalencia de VTLS oscila entre el 4 y el $35.6 \%{ }^{2}$. La sacralización se ha reportado en el 3 a $7 \%$ de la población y es más común en hombres, mientras que la lumbarización tiene una prevalencia entre el 1.7 y el 14\% y es más común en mujeres ${ }^{3}$. El SB se diagnostica en el 4 a $8 \%$ de los pacientes jóvenes de entre 30 y 40 años con dolor crónico de espalda ${ }^{1}$, y llega al $11.4 \%$ en menores de 30 años $^{4}$.

La etiología del dolor es multifactorial e incierta. Es secundario a alteraciones en la biomecánica por degeneración temprana discal o hernia por encima del nivel de las VTLS, cambios en las articulaciones facetarias contralaterales en casos de fusión unilateral, o cambios extraforaminales en el sitio de articulación del segmento de las VTLS al sacro o cresta ilíaca por la presencia de apófisis transversa más amplia ${ }^{1}$.

\section{PRESENTACIÓN DEL CASO}

Mujer de 37 años de edad, acude por dolor lumbar bajo de 4 años de evolución, no irradiado y que incrementa tras los esfuerzos físicos moderados. Al examen físico mostró sensibilidad a la palpación profunda sobre las articulaciones facetarias bilaterales, de predominio derecho, con prueba de elevación de pierna recta negativa. Se le solicitó una radiografía simple de columna lumbar anteroposterior y lateral (Fig. 1), donde se observa una leve rotoescoliosis dextroconvexa, lumbarización de S1 con megaapófisis transversa, la derecha articulada con el sacro y la izquierda aparentemente fusionada.
Además, en la placa lateral se observó hiperlordosis y leve anterolistesis de L4 sobre L5. Se decidió tratamiento conservador con antiinflamatorios y fisioterapia, sin embargo, al concluirlo, los síntomas persistieron, por lo que se decide realizar resonancia magnética (RM) simple de columna lumbar (Figs. 2 y 3), donde además de los hallazgos vistos en la radiografía se observó defecto de fusión de L5 con morfología en «mariposa», protrusión central del contenido discal con fisura anular concéntrica del disco L4-L5 e hipertrofia con leve sinovitis de las articulaciones facetarias. Posterior a los hallazgos de imagen, la paciente fue sometida a bloqueo anestésico de la pseudoarticulación transversa-sacra derecha. Actualmente la paciente se encuentra asintomática.

\section{DISCUSIÓN}

La columna vertebral y la médula espinal tienen un desarrollo temprano en la gestación, de manera secuencial y ajustada, en 6 fases separadas pero superpuestas ${ }^{5}$. Cualquier alteración en la secuencia normal puede llevar a variaciones en la anatomía estructural.

Las malformaciones vertebrales se han clasificado de acuerdo con la embriopatía subyacente en simples (con poca o ninguna consecuencia clínica) o complejas (con serias implicaciones estructurales y neurológicas). Las variaciones de desarrollo que conducen a vértebras de transición en las uniones toracolumbar y lumbosacra se encuentran en una categoría separada ${ }^{6}$. Estas variaciones se producen en la segunda fase (formación de somitas), y están determinadas por la interacción de varios genes (HOX 10 y HOX 11). La 

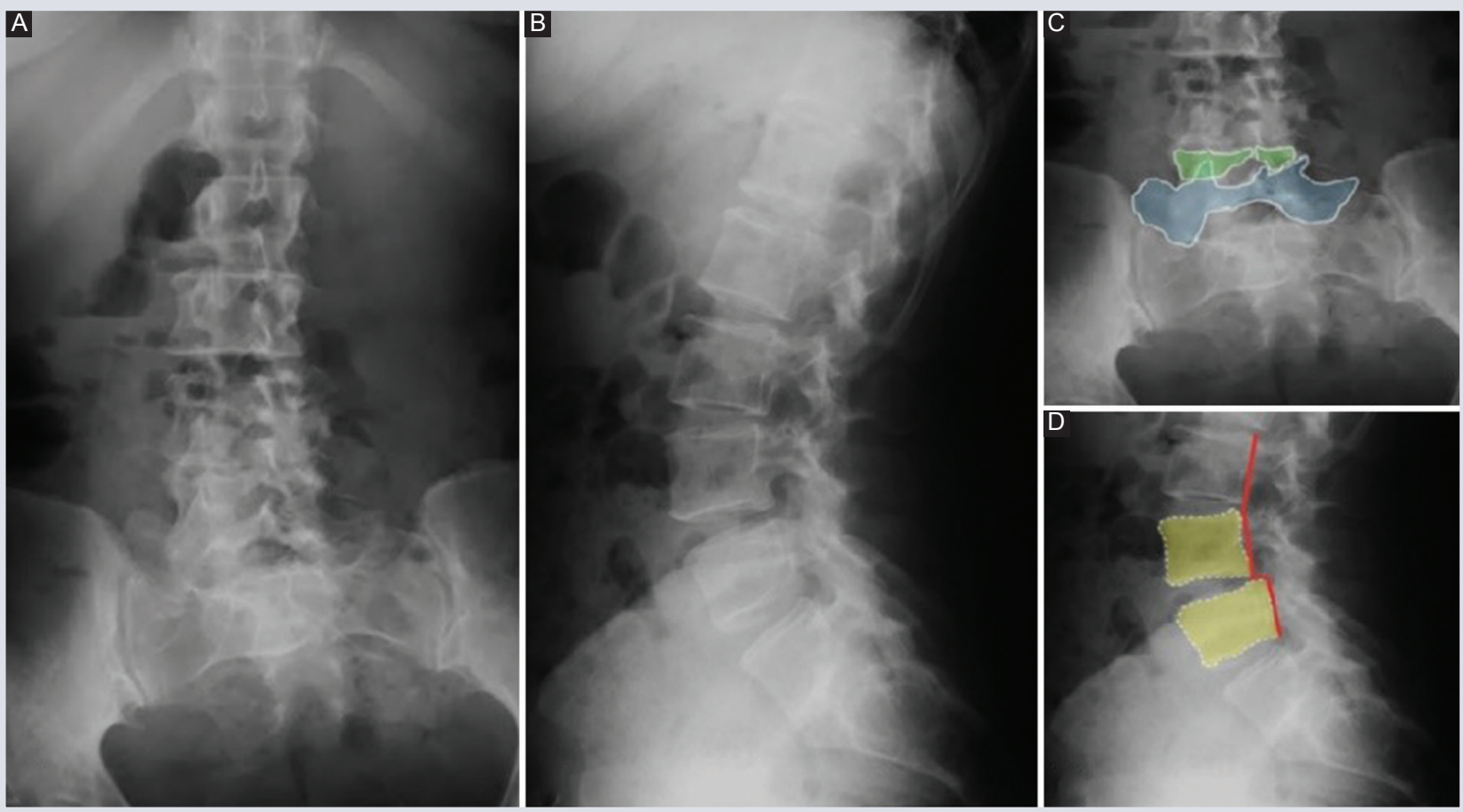

Figura 1. A: radiografía AP. B: radiografía lateral de la columna lumbar. A: leve rotoescoliosis dextroconvexa, lumbarización de S1 con megaapófisis transversas, la derecha articulada con el sacro y la izquierda aparentemente fusionada. B: hiperlordosis y anterolistesis de L4 sobre L5 grado I. C-D: esquema de los hallazgos relevantes. AP: anteroposterior.
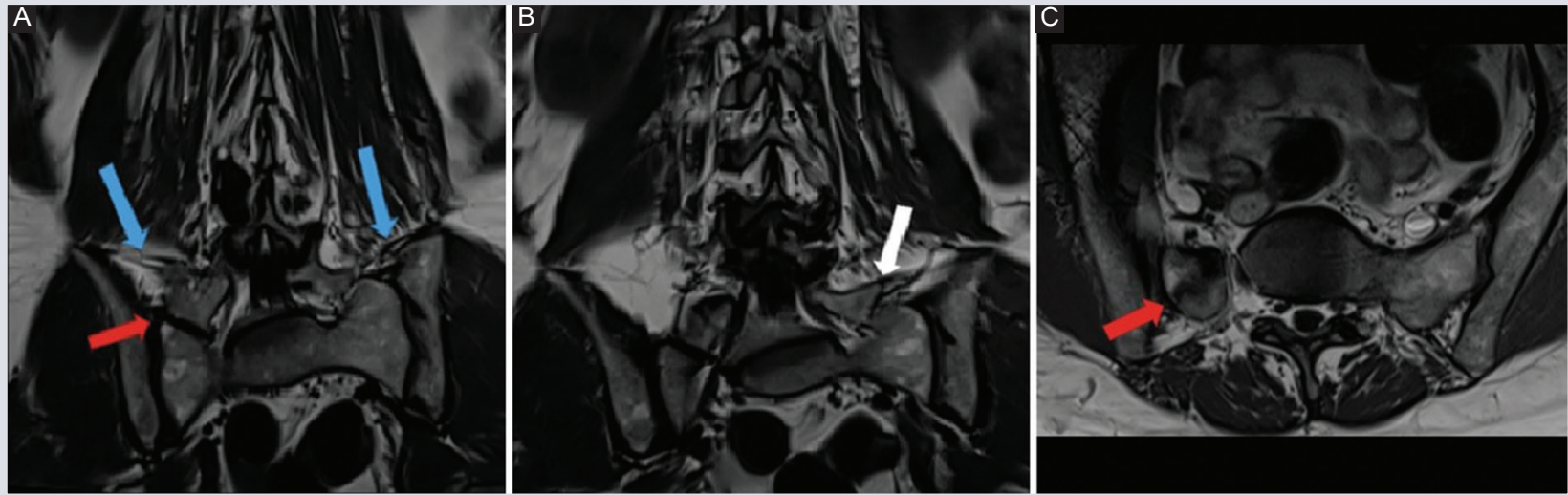

Figura 2. RM de columna lumbar potenciada en T1. A-B: imágenes en el plano coronal donde se observan los ligamentos iliolumbares (flechas azules), la pseudoarticulación de la megaapófisis transversa derecha al sacro (flecha roja), y la izquierda (flecha blanca) parcialmente fusionada en B (por lo que corresponde a una clasificación Castellvi IV). C: imagen en el plano axial donde se observa la articulación de la megaapófisis transversa derecha con el sacro (flecha roja).

expresión incorrecta de estos podría explicar la presencia de vértebras transicionales ${ }^{4}$.
Segmento transicional es aquel que conserva características parciales del segmento por 


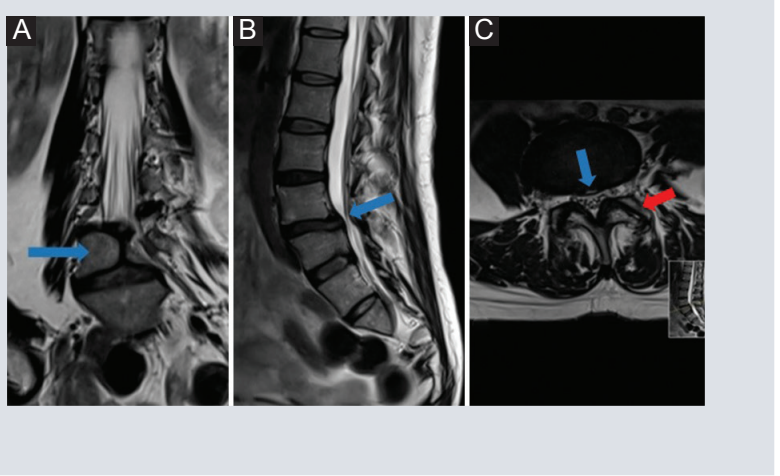

Figura 3. Resonancia magnética de columna lumbar. A: en el plano coronal. B: sagital. C: axial. Potenciadas en T2. A: defecto de fusión de L5, con morfología en "mariposa" (flecha azul). B: anterolistesis de L4 sobre L5 (flecha azul). C: protrusión central del disco con desgarro concéntrico del anillo fibroso en L4-L5 (flecha azul) e hipertrofia con sinovitis de las articulaciones facetarias (flecha roja).

encima y por debajo de él ${ }^{7}$. Se prefiere este término para evitar tener que decidir si la vértebra es una L5 sacralizada o una S1 lumbarizada, ya que no es posible saber qué tipo de vértebra representa sin ver toda la columna vertebral ${ }^{8}$. Una vértebra transicional completa produce anomalías en la numeración de los segmentos lumbar y sacro, con cuatro vértebras lumbares en caso de sacralización y seis en caso de lumbarización. Existen transiciones intermedias incompletas que han sido clasificadas en cuatro tipos por Castellvi, et al. (Fig. 4) $)^{9}$.

Para el diagnóstico se ha descrito la utilidad de la radiografía AP con ángulo craneal de $30^{\circ}$ (Ferguson) para identificar una VTLS y asociada a una proyección AP que incluya la unión toracolumbar permite la evaluación del nivel vertebral ${ }^{1,4,10}$. Sin embargo, características como costillas cortas en T12 dan la apariencia de seis vértebras lumbares, procesos transversales largos de L1 simulan cuatro vértebras lumbares. En ocasiones existe una treceava costilla ${ }^{8}$. Por lo que la situación ideal es contar con una imagen panorámica para poder numerar las vértebras.

La tomografía computarizada es la técnica de imagen primaria para describir trastornos de las estructuras óseas, y por tanto, la mejor técnica para la caracterización de VTLS, sin embargo, no es la técnica preferida para evaluar el dolor lumbar no traumático, por lo que suele ser un hallazgo incidental ${ }^{11}$.

En RM, la clasificación y numeración es más problemática debido a factores que incluyen imágenes limitadas dela unión toracolumbar ${ }^{10}$. En ausencia de imágenes coronales, hasta el $35.1 \%$ de los pacientes con VTLS pueden ser diagnosticados con segmentación normal ${ }^{4}$. Al comparar el recuento en imágenes de localizador de la columna lumbar en los planos sagital y coronal con el recuento caudal en imágenes adicionales sagitales de la región cervical-torácica, la concordancia es solo del $80 \%$ de los pacientes ${ }^{12}$.

Las imágenes sagitales en pacientes con VTLS muestran con frecuencia una curvatura lordótica acentuada, con una angulación lumbosacra $>39.8^{\circ}$ y una inclinación sacra $>$ $35.9^{\circ 4}$ (Fig. 5).

Se han descrito marcadores anatómicos para la identificación de segmento transicional en la RM, entre los que destacan el ligamento iliolumbar, el cual es uno de los tres ligamentos lumbopélvicos que estabilizan la pelvis, el sacro y la L5. En el 96\% de los casos se extiende del proceso transverso de L5 al ala ilíaca posterior y a la cresta del ilion ${ }^{4}$.

La bifurcación aórtica se localiza a nivel del cuerpo de L4 en un $83 \%$, y se encuentra en 


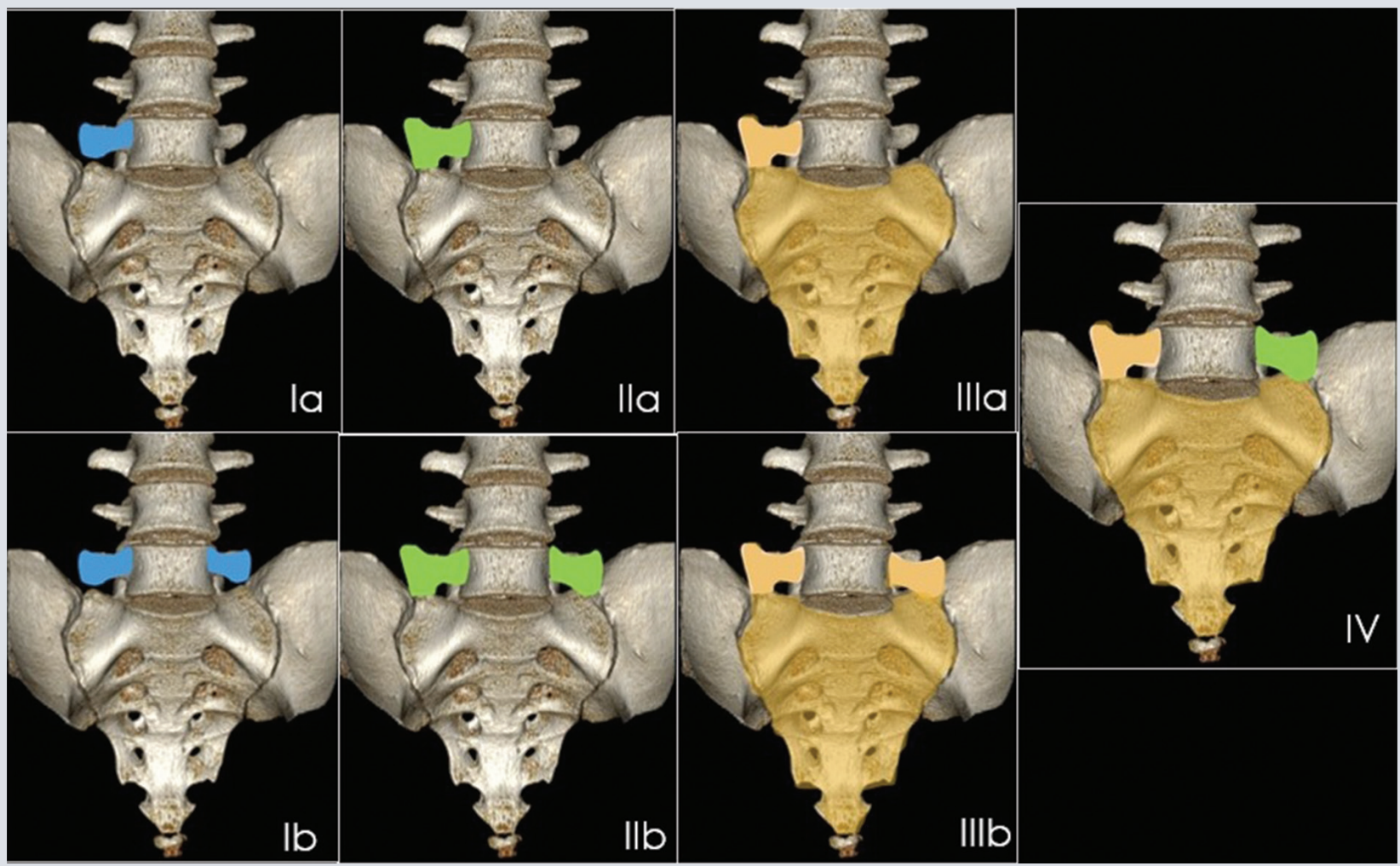

Figura 4. Esquema realizado con base en la descripción de Castellvi. Tipo I: Procesos transversales displásicos ( $\geq 19 \mathrm{~mm}$ craneocaudal), unilateral (Ia) o bilateral (Ib). Tipo II: Lumbarización/sacralización incompleta, unilateral (Ila) o bilateral (IIb), con una megaapófisis que tiene una pseudoarticulación con el ala sacra adyacente. Tipo III: Lumbarización/sacralización completa con fusión ósea de los procesos transversales al sacro, unilateral (IIla) o bilateral (IIIb). Tipo IV: Mixta (tipo II de un lado y tipo III en el lado contralateral).

posición caudal en caso de lumbarización de S1 y en posición cefálica en caso de sacralización $^{13}$. La arteria renal derecha generalmente se encuentra en el espacio del disco L1-L2, sin embargo, en el 25\% de los casos no se visualiza o está presente en otra ubicación ${ }^{10}$.

Se ha descrito una morfología «cuadrada» del segmento sacro superior cuando está lumbarizado, o un acuñamiento del segmento lumbar más bajo cuando está sacralizado. La apariencia «cuadrada» se define como la relación del diámetro AP del platillo vertebral superior al inferior $<1.37$ en las radiografías laterales $^{12} \mathrm{o}<1.1$ en la $\mathrm{RM}^{8}$.
La visualización por RM de la última articulación costotransversa en T12 se utiliza para identificar la VTLS, tiene una sensibilidad del $74.14 \%$ y una especificidad del $96.85 \%{ }^{14}$.

El origen del músculo psoas en caso de sacralización se origina en los niveles T12 o T12-L1 (95.7\%), similar al grupo de segmentación normal; en caso de lumbarización, en L1 o L1-L2 (66.7\%) ${ }^{14}$. En series de RM se encontró que la inserción proximal fue en L1 en el $94 \%$, por lo que algunos consideran que se trata de un hito confiable ${ }^{15}$.

El nervio L5 es una buena referencia anatómica, gracias a sus características: es el único 


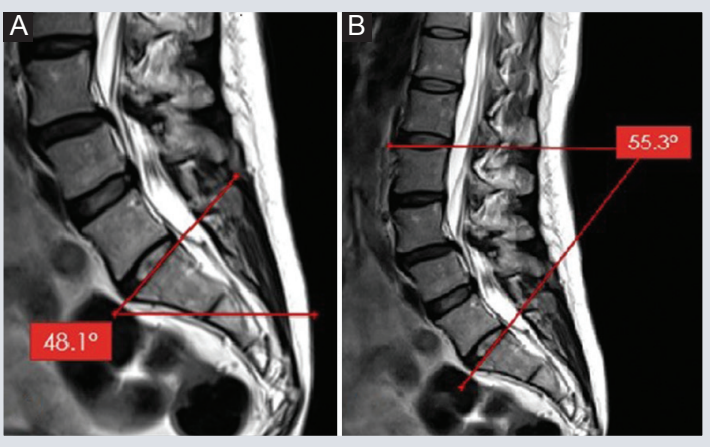

Figura 5. A: angulación lumbosacra, formada por una línea paralela a la superficie superior del sacro y una línea perpendicular al eje de la mesa en la resonancia magnética sagital en T2 (especificidad y sensibilidad: $80 \%$ ). B: inclinación sacra, formada por una línea paralela al platillo superior de L3 y una línea paralela a la superficie superior del sacro (sensibilidad: $80 \%$ y especificidad: $54 \%)^{4}$.

nervio lumbar sin ramificación proximal, recibe un fascículo posterior de L4 y, por lo general, tiene mayor calibre. De esta manera, si el primer nervio no dividido se rastrea hasta el primer cuerpo del sacro, se entenderá que existe sacralización de L5, mientras que si el primer nervio no dividido se rastrea hasta un cuerpo vertebral a dos niveles por encima del primer cuerpo sacro, se entenderá que existe lumbarización de $S 1^{16}$.

Otras referencias tienen un origen variable desde T12 a L2, por lo que muchos autores no recomiendan su utilización ${ }^{14}$.

Una VTLS altera la anatomía y la biomecánica normal de la columna vertebral. El sacro distribuye el peso de la parte superior del cuerpo hacia la articulación sacroilíaca. La capacidad para disipar la carga depende de su tamaño y área de superficie con la articulación sacroilíaca ${ }^{4}$. Los pacientes con VTLS tienen ligamentos iliolumbares más delgados y débiles, lo que lleva a una inestabilidad e hipermovilidad del segmento vertebral. La carga es absorbida por el proceso transversal fusionado protegiendo al disco, por lo que el segmento suprayacente debe soportar la tensión adicional. Esto podría conducir a una degeneración temprana del disco ${ }^{4,10}$.

En caso de VTLS unilateral, el lado que lleva la relación L5-S1 soporta mayor carga, lo que resulta en una inclinación lateral de la cresta ilíaca y una convexidad de una curva escoliótica hacia el lado de la articulación. La asimetría puede causar cambios degenerativos tempranos dentro de la articulación facetaria contralateral normal, dando lugar a dolor facetario $^{4}$.

Existe variación de los dermatomas lumbosacros cuando hay sacralización de $\mathrm{L} 5$, la raíz del nervio L4 cumple la función de la raíz del nervio L5, y, de manera similar, cuando está presente un S1 lumbarizado, la raíz del nervio S1 funciona como la raíz del nervio L5. La raíz de L5 funcional siempre se origina en el nivel móvil más bajo, por definición corresponde con aquel que posea espacio discal completamente formado, articulaciones facetarias bilaterales y procesos transversales no articulados al sacro o pelvis ${ }^{4,17}$. Conocer estas variaciones ayuda a explicar los síntomas radiculares de confusión.

La VTLS se ha asociado a estenosis extraforaminal entre el proceso transverso de la vértebra de transición y el ala sacra, que causa atrapamiento de la raíz nerviosa y radiculopatía. Este hallazgo ha sido denominado «síndrome de far-out» ${ }^{12}$.

El tratamiento inicial es sintomático e incluye una combinación de AINE y terapia física de 
rehabilitación, sin embargo, no existe un consenso ${ }^{4}$. La inyección de esteroides o anestésicos locales en el sitio de pseudoarticulación puede ser diagnóstica y proporcionar alivio inmediato, aunque temporal del dolor. Otra opción mínimamente invasiva podría ser la denervación mediante radiofrecuencia, la cual da un alivio a largo plazo ${ }^{4-10}$. Si el bloqueo de la articulación anómala no proporciona alivio del dolor, se pueden considerar otras causas asociadas de dolor lumbar. Se considera la resección quirúrgica parcial de la megaapófisis una opción en pacientes seleccionados, sin embargo, los resultados del tratamiento quirúrgico son solo un poco mejores que el tratamiento conservador ${ }^{17}$.

\section{CONCLUSIONES}

Debido a que ciertas anomalías vertebrales pueden llegar a ser sintomáticas, es importante la interpretación precisa de las imágenes, lo que requiere el conocimiento de la morfología vertebral normal, anormal y variantes. Esto influirá en el manejo adecuado para cada entidad. La identificación precisa de segmentos vertebrales transicionales y del nivel vertebral puede ser crítica, principalmente en cirugía. Debido a que ningún punto de referencia es sistemáticamente confiable, se sugiere hacer una declaración explícita en el informe de imágenes de RM de la columna lumbar con respecto a cómo se determinó la unión lumbosacra.

El síndrome de Bertolotti debe considerarse en el diagnóstico diferencial del dolor lumbar, especialmente en pacientes jóvenes, sin embargo, debe basarse en una historia clínica adecuada, estudios de imagen y el alivio del dolor tras la inyección en el sitio de dolor con esteroides y/o anestésicos.

\section{FINANCIAMIENTO}

Este trabajo no requirió financiamiento.

\section{CONFLICTO DE INTERESES}

Los autores declaran no tener ningún conflicto de intereses.

\section{RESPONSABILIDADES ÉTICAS}

Protección de personas y animales. Los autores declaran que para esta investigación no se han realizado experimentos en seres humanos ni en animales.

Confidencialidad de los datos. Los autores declaran que han seguido los protocolos de su centro de trabajo sobre la publicación de datos de pacientes.

Derecho a la privacidad y consentimiento informado. Los autores han obtenido el consentimiento informado de los pacientes y/o sujetos referidos en el artículo. Este documento obra en poder del autor de correspondencia.

\section{BIBLIOGRAFÍA}

1. Kapetanakis S, Chaniotakis C, Paraskevopoulos C, Pavlidis P. An Unusual Case Report of Bertolotti's Syndrome: Extraforaminal Stenosis and L5 Unilateral Root Compression (Castellvi Type III an LSTV). J Orthop Case Reports. 2017;7(3):9-12.

2. Shaikh A, Khan SA, Hussain M, Soomro S, Adel H, Adil SO, et al. Prevalence of lumbosacral transitional vertebra in individuals with low back pain: Evaluation using plain radiography and magnetic resonance imaging. Asian Spine J. 2017;11(6):892-7. 
3. French HD, Somasundaram AJ, Schaefer N R, Laherty RW. Lumbosacral transitional vertebrae and its prevalence in the Australian population. French. Global Spine Journal. 2014;4(4):229-32.

4. Jancuska J, Spivak J, Bendo J. A Review of SymptomaticLumbosacral Transitional Vertebrae: Bertolotti's Syndrome. Int J Spine Surg. 2015;9:42.

5. Dias MS. Normal and Abnormal Development of the Spine. Neurosurg Clin N Am. 2007;18(3):415-29.

6. Chaturvedi A, Klionsky NB, Nadarajah U, Chaturvedi A, Meyers SP. Malformed vertebrae: a clinical and imaging review. Insights Imaging. 2018;9(3):343-55.

7. Carrino JA, Campbell PD, Lin DC, Morrison WB, Schweitzer ME, Flanders AE, et al. Effect of Spinal Segment Variants on Numbering Vertebral Levels at Lumbar MR Imaging. Radiology. 2011;259(1):196-202.

8. Paik NC, Lim CS, Jang HS. Numeric and Morphological Verification of Lumbosacral Segments in 8280 Consecutive Patients. Spine (Phila Pa 1976). 2013;38(10):E573-8.

9. Castellvi AE, Goldstein LA, Chan DP. Lumbosacral transitional vertebrae and their relationship with lumbar extradural defects. Spine (Phila Pa 1976). 1984;9:493-5.

10. Konin GP, Walz DM. Lumbosacral transitional vertebrae: Classification, imaging findings, and clinical relevance. AJNR Am J Neuroradiol. 2010;31(10):1778-86.
11. Van Rijn RM, Wassenaar M, Verhagen AP, Ostelo RW, Ginai AZ, de Boer MR, et. al. Computed tomography for the diagnosis of lumbar spinal pathology in adult patients with low back pain or sciatica: a diagnostic systematic review. Eur Spine J. 2012;21(2):228-39.

12. Hughes RJ, Saifuddin A. Imaging of lumbosacral transitional vertebrae. Clinical Radiol. 2004;59(11):984-91.

13. Hughes RJ, Saifuddin A. Numbering of Lumbosacral Transitional Vertebrae on MRI: Role of the Iliolumbar Ligaments. AJR Am J Roentgenol. 2006;187(1):W59-65.

14. Jagannathan D, Indiran V, Hithaya F, Alamelu M, Padmanaban S. Role of anatomical landmarks in identifying normal and transitional vertebra in lumbar spine magnetic resonance imaging. Asian Spine J. 2017;11(3):365-79.

15. Ropars F, Mesrar J, Ognard J, Querellou S, Rousset J, Garetier M. Psoas proximal insertion as a simple and reliable landmark for numbering lumbar vertebrae on MRI of the lumbar spine. Eur Radiol. 2019;29(5):2608-15.

16. Peckham ME, Hutchins TA, Stilwill SE, Mills MK, Morrissey BJ, Joiner EAR, et al. Localizing the L5 Vertebra Using Nerve Morphology on MRI: An Accurate and Reliable Technique. AJNR Am J Neuroradiol. 2017;38(10):2008-14.

17. Jadon A. Bertolotti's Syndrome and Its Management; Pain Physician Perspective -A Brief Review. Clin Surg. 2017;2:1737. 